\title{
THE STAR FORMATION HISTORY OF BCGs TO $z=1.8$ FROM THE SpARCS/SWIRE SURVEY: EVIDENCE FOR SIGNIFICANT IN SITU STAR FORMATION AT HIGH REDSHIFT
}

\author{
Tracy M. A. Webb ${ }^{1}$, Adam Muzzin ${ }^{2}$, Allison Noble ${ }^{3}$, Nina Bonaventura ${ }^{1}$, James Geach $^{4}$, Yashar Hezevah ${ }^{5}$, \\ Chris Lidman $_{1}^{6}$, Gillian Wilson ${ }^{7}$, H. K. C. Yee ${ }^{3}$, Jason Surace ${ }^{8}$, and David Shupe ${ }^{9}$ \\ ${ }^{1}$ McGill University, 3600 rue University, Montreal, QC H3A 2T8, Canada \\ ${ }^{2}$ Leiden Observatory, University of Leiden, P.O. Box 9514, 2300 RA Leiden, The Netherlands \\ ${ }^{3}$ Department of Astronomy and Astrophysics, University of Toronto, 50 St. George Street, Toronto, ON M5S 3H4, Canada \\ ${ }^{4}$ Centre for Astrophysics Research, University of Hertfordshire, Hatfield, Hertfordshire AL109AB, UK \\ ${ }^{5}$ Kavli Institue for Particle Physics and Cosmology, Stanford University, 452 Lomita Mall, Stanford, CA 94305-4085, USA \\ ${ }^{6}$ Australian Astronomical Observatory, P.O. Box 915, North Ryde, NSW 1670, Australia \\ ${ }^{7}$ Department of Physics and Astronomy, University of California, Riverside, CA 92521, USA \\ ${ }^{8}$ Spitzer Science Center, California Institute of Technology, M/S 314-6, Pasadena, CA 91125, USA \\ 9 NASA Herschel Science Center, IPAC, 770 South Wilson Avenue, Pasadena, CA 91125, USA \\ Received 2015 March 13; accepted 2015 August 19; published 2015 November 20
}

\begin{abstract}
We present the results of an MIPS-24 $\mu \mathrm{m}$ study of the brightest cluster galaxies (BCGs) of 535 high-redshift galaxy clusters. The clusters are drawn from the Spitzer Adaptation of the Red-Sequence Cluster Survey, which effectively provides a sample selected on total stellar mass, over $0.2<z<1.8$ within the Spitzer Wide-Area Infrared Extragalactic (SWIRE) Survey fields. Twenty percent, or 106 clusters, have spectroscopically confirmed redshifts, and the rest have redshifts estimated from the color of their red sequence. A comparison with the public SWIRE images detects 125 individual BCGs at $24 \mu \mathrm{m} \gtrsim 100 \mu \mathrm{Jy}$, or $23 \%$. The luminosity-limited detection rate of BCGs in similar richness clusters $\left(N_{\text {gal }}>12\right)$ increases rapidly with redshift. Above $z \sim 1$, an average of $\sim 20 \%$ of the sample have $24 \mu \mathrm{m}$ inferred infrared luminosities of $L_{\mathrm{IR}}>10^{12} L_{\odot}$, while the fraction below $z \sim 1$ exhibiting such luminosities is $<1 \%$. The Spitzer-IRAC colors indicate the bulk of the $24 \mu \mathrm{m}$ detected population is predominantly powered by star formation, with only $7 / 125$ galaxies lying within the color region inhabited by active galactic nuclei (AGNs). Simple arguments limit the star formation activity to several hundred million years and this may therefore be indicative of the timescale for AGN feedback to halt the star formation. Below redshift $z$ $\sim 1$, there is not enough star formation to significantly contribute to the overall stellar mass of the BCG population, and therefore BCG growth is likely dominated by dry mergers. Above $z \sim 1$, however, the inferred star formation would double the stellar mass of the BCGs and is comparable to the mass assembly predicted by simulations through dry mergers. We cannot yet constrain the process driving the star formation for the overall sample, though a single object studied in detail is consistent with a gas-rich merger.
\end{abstract}

Key words: galaxies: clusters: general - galaxies: evolution - galaxies: formation - galaxies: star formation

\section{INTRODUCTION}

At the center of most local galaxy clusters lies a single massive galaxy. These so-called brightest cluster galaxies (BCGs) are the most massive galaxies in the universe today. They do not appear, however, to simply be the extremes of the local galaxy mass function, but are separate beasts, with luminosities, metallicities, and surface brightness profiles that are unique and consistent across the population (Oemler 1976; Tremain \& Richstone 1977; Dressler 1978; Schombert 1986). It seems likely that the formation of BCGs is tied to the overall growth of their parent clusters through the physics of gas cooling in the most massive dark matter halos and their hierarchical accretion of the general field galaxy population.

Nevertheless, we know rather little about the formation histories of BCGs. Recent measurements by Lidman et al. (2012) show that BCGs have increased their stellar mass by $2 \times$ since $z \sim 1$ (cf. Collins 2009; Stott et al. 2011). These authors find the growth is driven by dry accretion of satellite galaxies, in line with semi-analytic models (Bower et al. 2006; De Lucia \& Blaizot 2007; Guo 2011). The accreted systems are gas poor, and no new star formation is induced during the merger process. In this picture most of the stellar mass is formed at very high redshifts $(z \sim 5)$ within multiple galaxies through low levels of star formation, and the BCG slowly acquires its identity through the conglomeration of previously assembled pieces. On the other hand, several moderate redshift BCGs $(z<0.6)$ exhibit signs of star formation $\left(10-1000 M_{\odot} \mathrm{yr}^{-1}\right)$ and/or contain large amounts of molecular gas (Johnstone et al. 1987; Allen et al. 1992; McNamara \& O'Connell 1992; Cardiel et al. 1998; Crawford et al. 1999; Edge 2001; Wilman et al. 2006; Edwards et al. 2007; O'dea et al. 2010; Donahue et al. 2011; McDonald et al. 2012; Rawle et al. 2012), indicating the scenario is not so simple. In many cases, the star formation rates (SFRs) are correlated with the gas cooling time, and indeed star formation has been primarily seen in cool-core clusters. It is not yet clear how important this star formation is to the overall growth of BCGs as a population nor how it might relate to similar processes at very high redshift that are posited by the models.

Star formation fed by rapid gas deposition and continual dry galaxy accretion are two very different scenarios of BCG evolution, and each have implications for the physics of halo collapse and growth. They are not mutually exclusive since both are transient events regulated by different processes and may occur at different times in the history of a galaxy. BCG galaxy accretion is an ongoing, though sporadic, phenomenon, fed by the continual infall of field galaxies into the cluster potential, which are stripped of much of their interstellar 
medium (ISM) during their descent into the center of the cluster. Cooling flows, and the resulting central starburst, likely have defined duty cycles that are governed by still unclear heating and cooling mechanisms (McNamara \& Nulsen 2007; Rafferty et al. 2008; Voit et al. 2008).

Studies of galaxy clusters and their central galaxies suffer from observational bias and, until recently, small sample sizes. Much of the work on central star formation has been carried out on X-ray-selected clusters that may preferentially select cooling flow clusters. Quite recently, large samples of SunyaevZeldovich clusters have become available through facilities such as the South Pole Telescope (e.g. McDonald et al. 2013) that also select clusters through observations (though indirect) of their intercluster medium. Here, we investigate the observed IR properties of BCGs using a large sample of clusters from the Spitzer Adaptation of the Red-Sequence Cluster Survey (SpARCS), an optical/NIR galaxy-selected cluster survey, designed to discover clusters to $z \sim 2$ (Muzzin et al. 2009; Wilson et al. 2009). While an important expansion into new parameter space, this approach also introduces new limitations and challenges. With a large sample of galaxy clusters, considerably less is known about each individual system, and the conclusions are generally statistical. It is necessary to automate some aspects of the analysis that increase the risk of sample contamination. Thus, we present the results of this work with the reminder that ongoing studies, such as high-resolution imaging and additional spectroscopy, are required to better calibrate some of the assumptions made here and relate the findings to those of X-ray- and SZ-selected samples.

The paper is outlined as follows. In Section 2, we introduce the SpARCS cluster sample. In Section 3, we outline our BCG identification algorithm and the IR analysis using the Spitzer Wide-Area Infrared Extragalactic (SWIRE) data. In Section 4, we discuss the origin of the IR emission and the change in the IR properties of BCGs with redshift. Section 5 finishes with a discussion of the implications of Section 4, and we present our conclusions in Section 6. Standard cosmology $\left(H_{\circ}=70 \mathrm{~km} \mathrm{~s}^{-1} \mathrm{Mpc}^{-1} ; \Omega_{\text {matter }}=0.3 ; \Omega_{\Lambda}=0.7\right)$ is adopted throughout.

\section{DATA AND OBSERVATIONS}

\subsection{The SpARCS}

The SpARCS is a deep $z^{\prime}$-band imaging survey covering the Spitzer SWIRE Legacy fields, designed to assemble a large homogeneously selected sample of massive clusters to $z \sim 2$ (Muzzin et al. 2009, 2012; Wilson et al. 2009; Demarco et al. 2010). It employs an infrared adaptation of the cluster redsequence method (Gladders \& Yee 2005): using the $z^{\prime}-$ $3.6 \mu \mathrm{m}$ color, which spans the $4000 \AA$ break at $z>1$, it locates overdensities of red-sequence galaxies that trace massive galaxy clusters. This simultaneously provides a reliable redshift estimate for the cluster through the color fit to the red sequence, discussed in more detail in the Section 3.

SpARCS uses the public SWIRE images and catalogs that provide photometry measurements at $3.6,4.5,5.8$, and $8.0 \mu \mathrm{m}$ from the IRAC instrument and $24 \mu \mathrm{m}$ with the MIPS camera (Lonsdale et al. 2004; D. Shupe et al. 2015, private communication). SWIRE contains northern and southern sky coverage; however, to ensure uniform ancillary data (described below), we limit this work to the 34 square degrees of the
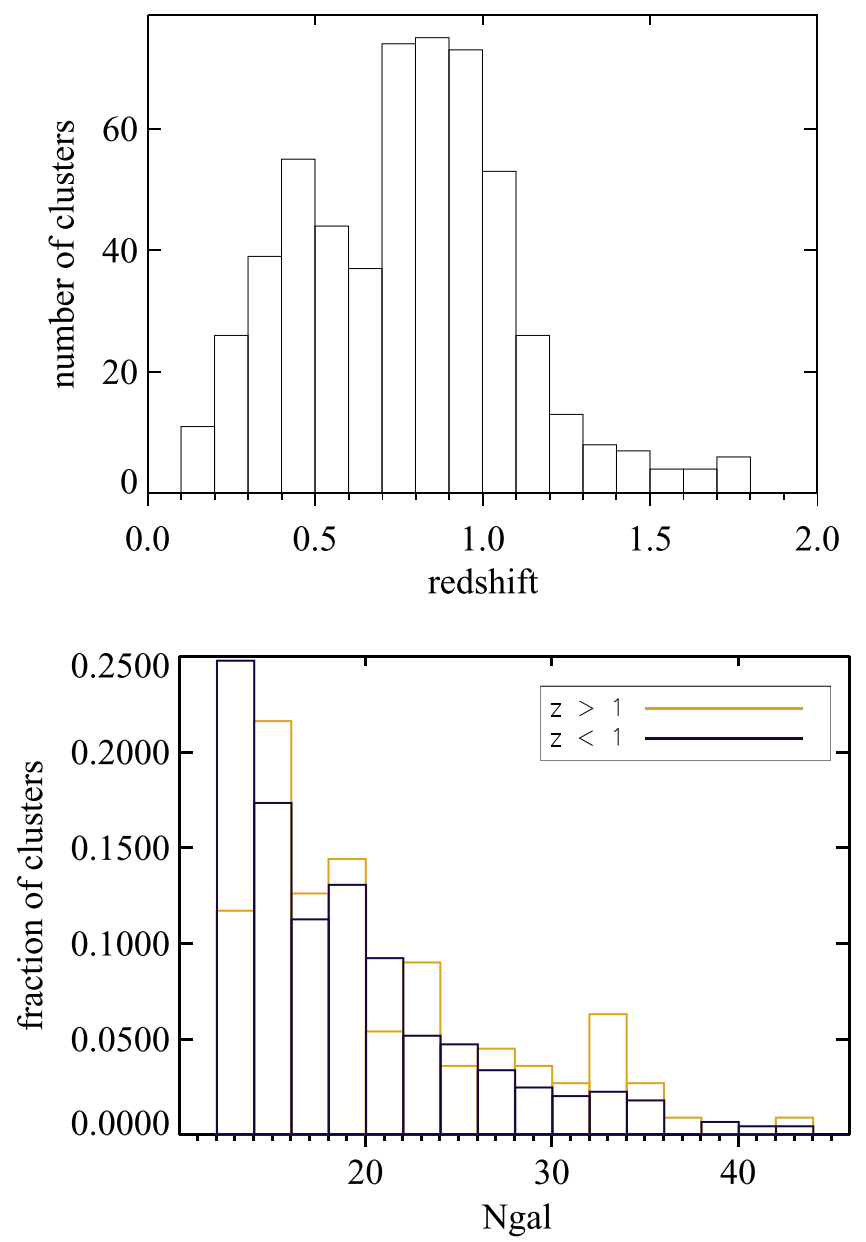

Figure 1. Top: the redshift distribution of the SpARCS cluster sample used here. Bottom: the richness distribution (parameterized by $N_{\text {gal }}$ ) of the $z<1$ clusters (black) and the $z>1$ clusters (orange). Although fewer clusters are found at higher redshift, there is no significant difference in the richness distribution between the two redshift bins.

northern fields (ELAIS-N1/2, XMM-LSS, Lockman) and further require uniform coverage in IRAC 3.6 and $4.5 \mu \mathrm{m}$.

The parent sample is limited by the detection reliability of the red-sequence overdensity and the measured NIR richness. Galaxy clusters must have a "flux" of at least 4 in overdensity in the detection map-a criteria similar to that of Hildebrandt et al. (2011) — and have a measured richness of $N_{\text {gal }}>12 . N_{\text {gal }}$ is defined as the number of background-subtracted galaxies within an aperture of $500 \mathrm{kpc}$ above a limit of $M_{3.6 \mu \mathrm{m}}^{*}+1$. These criteria ensure a richness-limited sample of high signalto-noise detections (Figure 1). To place the richness in physical context, we note that $N_{\text {gal }}=12$ corresponds to $M_{200} \sim$ $1 \times 10^{14} M_{\odot}$ (Wen et al. 2010, 2012; Capozzi et al. 2012; Andreon \& Congdon 2014). The sample of 559 galaxies is further culled as discussed in Section 3.

\section{ANALYSIS}

\subsection{BCG Identification}

Given the large number of clusters (a final sample of 535, 106 confirmed and 429 candidates) within our sample, we automate the BCG identification algorithm. We select the brightest galaxy in the IRAC $3.6 \mu \mathrm{m}$ channel whose color is within $\pm 0.5 \mathrm{mag}$ of the predicted red sequence. The location 


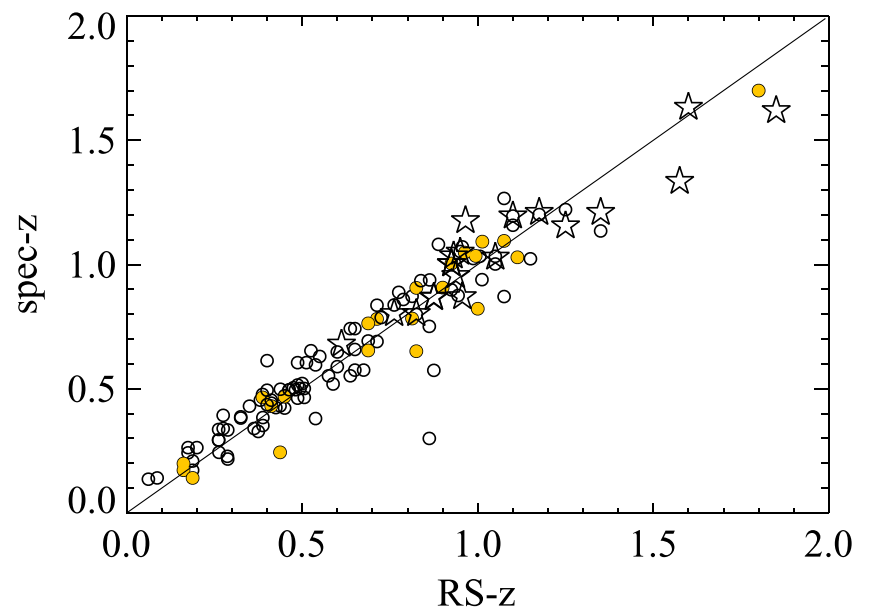

Figure 2. Comparison of the cluster redshift, estimated through the location of the red sequence, and the BCG spectroscopic redshift (open circles) or cluster spectroscopic (open stars) redshift where available (Wenger et al. 2000; Rowan-Robinson et al. 2008, 2013; Coil et al. 2011; Muzzin et al. 2012; Cool et al. 2013; Fang et al. 2015). The solid line denotes a 1:1 correspondence. The BCG catalog has been cleaned by eye to remove all obvious low-redshift galaxy misidentifications. Solid orange points show BCGs with $24 \mu \mathrm{m}$ counterparts (Section 3.3), and these do not have a systematically different redshift offset than the $24 \mu \mathrm{m}$ non-detected population. The overall scatter in red-sequence redshift is $\sigma=0.10$, using 106 spectroscopic redshifts.

red sequence is defined by the $z^{\prime}-3.6 \mu \mathrm{m}$ color models of Bruzual \& Charlot (2003), following Muzzin et al. (2009). Using clusters in common we check this against the smaller, but more detailed, study of high-redshift BCGs of Lidman et al. (2012) and find that they select the same objects. This method is, however, susceptible to contamination by foreground dusty galaxies which are likely to be bright at $24 \mu \mathrm{m}$ and may have colors sufficiently reddened so as to be consistent with the red sequence at higher redshift. Since the goal of this work is to study the infrared properties of the BCGs, it is imperative that the frequency of such interlopers be quantified and the misidentified BCGs removed from the analysis.

We identify candidate interlopers through visual inspection of the galaxy morphology. We use the Sloan Digital Sky Survey (SDSS) as it provides uniform imaging over all four fields. We identify galaxies with obvious spiral structure and remove these from the analysis. We perform a secondary check using the the deeper imaging of the Canada-France-Hawaii Telescope Legacy Survey in the XMM-LSS field, but do not identify any additional interlopers. In total, 24/559 galaxies are removed, leaving a final sample of 535 BCGs.

We then compare the spectroscopic redshift of the candidate BCG when available with the red-sequence-estimated redshift of the cluster. This comparison is shown in Figure 2 and includes 106 spectroscopic redshifts assembled from SDSS, our own work (Muzzin et al. 2012), the OzDES survey (Fang et al. 2015), PRIMUS (Coil et al. 2011; Cool et al. 2013), and the literature (Wenger et al. 2000; Rowan-Robinson et al. 2008, 2013). A systematic offset of $z(\mathrm{RS})-0.15$ has been applied to the entire sample. This offset was empirically determined from these 106 redshifts to correct for differences between the model and measured colors of the red sequence. We are, as of yet, unsure of the source of this offset, but this will be further explored in A. Muzzin et al. (2015, in preparation). As can be seen in Figure 2, there is excellent agreement between redshifts, and no additional galaxies are removed based on redshift disagreements. Although there are several BCGs whose RS redshift estimates are low compared to the overall scatter within the population, there is only one catastrophic outlier $(|\Delta z|>0.5)$. This small offset may reflect a tendency of the RS method to occasionally overestimate the redshift, rather than a misidentification of the BCG. After all interlopers are removed, we are left with a final sample of 535 BCGs. The richness of their parent clusters span $N_{\mathrm{gal}}=12-40$ at all redshifts, and the redshift distribution is shown in Figure 1.

\subsection{Caveats on Identification of the $B C G$}

This method of BCG identification has two additional biases. First, it is biased against BCGs with unusual colors-i.e., colors that are inconsistent with the red sequence. Thus, if a BCG were exceptionally blue or red, for example, due to star formation (unobscured or obscured), it would be passed over by the algorithm, and instead the second brightest red-sequence galaxy in the cluster would be chosen (maintaining the redshift agreement, but technically selecting the wrong galaxy). In contrast to foreground contamination, which adds spurious star-forming galaxies to the sample, this effect may remove real star-forming BCGs from the sample.

Second, the sample could be contaminated by late-stage mergers that are unresolved in the IRAC $3.6 \mu \mathrm{m}$ imaging (with a point response function of 1".8). Such systems, which are actually two galaxies but still cluster members, may appear as the brightest galaxy within the cluster when their light is combined. This latter effect would be more important at higher redshift where the apparent separation is smallest. We see no signs of this in the optical imaging, but higher-resolution observations of a significant fraction of the sample are required to confirm this.

Aside from the practical difficulties in identifying the BCG of a distant galaxy cluster, we must also consider the evolving definition of the BCG itself. $N$-body and SAMS (De Lucia \& Blaizot 2007) indicate that BCGs do not exist as a single identifiable object above $z \sim 0.7$, and this is supported by some, but not all, observations of distant clusters (e.g., Santos et al. 2009; Webb et al. 2015). In these models, where the BCG assembles through dry mergers and galaxy cannibalism, the stellar mass that will eventually form the BCG is distributed among more than one galaxy. Even so, these galaxies will still be among the most massive systems in the cluster, and our algorithm would select the brightest of them. Thus, our approach may be more accurately described as identifying one of the progenitors of the BCG galaxy. An investigation of the uniqueness of BCG galaxies at high redshift is possible with these data, but beyond the scope of this paper, and will be the focus of later work by our group.

\section{3. $24 \mu \mathrm{m}$ Detected BCGs}

The BCG catalog was cross-referenced with the SWIRE IRAC-24 $\mu \mathrm{m}$ imaging catalogs, with an MIPS-BCG search radius of $3^{\prime \prime}$, to account for the uncertainty in the MIPS positions due to the $6^{\prime \prime}$ point response function. Table 1 lists the number of $24 \mu \mathrm{m}$ detected BCGs in each field to the depth of the SWIRE images $(\sim 150 \mu \mathrm{Jy})$, with a total of 125 detected systems, or $\sim 23 \%$ of the galaxies. Table 2 provides the coordinates, redshifts, and $24 \mu \mathrm{m}$ flux measurements of these systems. 
Table 1

Information on Cluster Sample and Observations

\begin{tabular}{|c|c|c|c|}
\hline SWIRE Field & $\begin{array}{l}\text { Number } \\
\text { of BCGs } \\
\text { in Sample }\end{array}$ & $\begin{array}{c}\begin{array}{c}\text { Number } \\
\text { of BCGs }\end{array} \\
\text { with } S_{24 \mu \mathrm{m}}>100 \mu \mathrm{Jy}\end{array}$ & $\begin{array}{l}\text { Expected } \\
\text { Spurious } \\
\text { Matches }\end{array}$ \\
\hline XMM-LSS & 124 & 29 & 2 \\
\hline Lockman & 202 & 43 & 2 \\
\hline ELAIS-N1 & 145 & 36 & 2 \\
\hline ELAIS-N2 & 64 & 17 & 1 \\
\hline total & 535 & 125 & 7 \\
\hline
\end{tabular}

Note.

${ }^{a}$ With the cluster richness $N_{\text {gal }}>12$.

\subsubsection{Spurious Alignments}

We estimate the number of spurious spatial coincidences through simple Monte Carlo simulations. Using the $24 \mu \mathrm{m}$ catalog, we repeat our BCG matching, but with random positions in place of the BCG locations. The number of expected spurious detections are listed for each field in Table 1 and indicate a spurious $\mathrm{BCG}-24 \mu \mathrm{m}$ contamination rate of $\sim 1 \%$ - much lower than our detection rate. We therefore do not attempt to correct for it in further analysis.

\subsubsection{Physical Misidentifications: Gravitationally Lensed Systems and Close Neighbors}

We classify as physical misidentification cases where the IR emission is not associated with the BCG itself, but with an object that is physically related to the BCG. Here, we consider gravitational lensing and close neighbors.

Strong gravitational lensing from the galaxy cluster potential and/or the central massive galaxy can magnify background IR galaxies along the line of sight to the cluster centers. If the offset between the lensed object and the BCG is less than $\sim 3^{\prime \prime}$, the IR flux will be confused within the Spitzer-MIPS beam and erroneously assigned to the BCG through the matching procedure.

To search for gravitational lenses within the SpARCS-BCG sample, we turn to archival or public Hubble Space Telescope (HST) imaging. Very few (10) of the sample presented here have been covered in past surveys, but that number increases if we include the less rich (but still meeting the significance criterion of Section 2.1) systems in SpARCS. In doing so, we find one example of a tight $\left(\sim 2^{\prime \prime}\right)$ lensing system in one cluster (Figure 3$)$. If this rate $(\sim 10 \%)$ is representative of the entire sample, lensing would indeed be an important source of contamination. However, this object is a previously known system that was first discovered as a strong galaxy-galaxy lens by Geach et al. (2007) and later imaged in a UDS field of the CANDELS survey (Grogin et al. 2011; Koekemoer et al. 2011). Its HST coverage may not be by chance, and therefore this rate may not be representative of the lensing rate.

To gauge if this lensing rate is at all reasonable, given what we know about the systems, we ran lensing simulations following the method of Hezaveh et al. (2012). We find small radius lensing rates of only $\sim 1 \%$, which are too low to account for the IR detection rates of $23 \%$; moreover, the rate does not increase with redshift. While the lensing rates can be fine-tuned by altering the simulations (for example, by tuning the ellipticity or BCG-cluster alignments), we conclude that lensing is not a major contaminant of our sample.

A second possible source of contamination that is physically interesting is the presence of close neighbors to the BCG. By this, we refer to physical neighbors rather than line-of-sight associations that would be accounted for in our Monte Carlo simulations of spurious detections. These galaxies need to be close enough to be confused within a single MIPS $24 \mu \mathrm{m}$ PRF, but separated into two objects in IRAC $3.6 \mu \mathrm{m}$. At such close separation, the objects will likely be interacting with the BCGs and thus since the two galaxies in a sense form a single system that includes the BCG, they modify, but do not negate, the interpretation of our results. To assess this issue, we have compared the frequency of close galaxy neighbors in the $24 \mu \mathrm{m}$ detected and undetected samples using the full IRAC SWIRE galaxy catalog and see no difference between the two; however, a proper assessment of this effect requires higherresolution imaging so that very close neighbors can be revealed.

\section{RESULTS}

\subsection{MIR Diagnostics of the Energy Source: Active Galactic Nucleus versus Star Formation}

In Figure 4 we show the 4-channel IRAC colors for the $24 \mu \mathrm{m}$ detected and the $24 \mu \mathrm{m}$ undetected BCGs. The IRAC color plot is frequently invoked as a tool to discriminate among active galactic nucleus (AGN; continuum-dominated), starforming (polycyclic aromatic hydrocarbon; PAH-dominated), and passive (stellar-dominated) galaxy systems (Lacy et al. 2004, 2007; Sajina et al. 2005; Stern et al. 2005; Donley et al. 2012). In Figure 4, we show the divisions suggested by Sajina et al. (2005; 1: low- $z$ PAH; 2 and 3: mid-/high- $z$ PAH and stellar continuum; 4: high- $z$ PAH and all- $z$ AGN) to statistically separate these populations as well as the more recent, and tighter, AGN selection criteria of Donley et al. (2012).

The IR-faint BCGs lie almost entirely within regions 2 and 3, indicating that they are truly passive systems, or higher-redshift star-forming galaxies with SFRs too low to be detected in SWIRE. The low-redshift star-forming region is relatively empty, containing only one IR-undetected and seven IRdetected systems. The cluster redshifts for these systems are $z<0.6$, in agreement with the expected low redshifts. The bulk $(\sim 75 \%)$ of the IR-detected objects lie within regions 1,2 , and 3 with higher-redshift systems preferring region 3, in agreement with the interpretation that their IR emission is star formation dominated. Region 4 contains only (bar one object) IR-detected systems. In this region, we find a combination of high-redshift star formers and AGNs. Indeed, many of these systems form an extension of the colors of regions 2 and 3, suggesting they are also dominated by starbursts.

Subsequent analysis identify as AGNs only those seven systems that lie within the updated constraints of Donley et al. (2012), but otherwise include BCGs in region 4. An upper limit to the contamination from AGNs to the MIPS detections can be obtained by considering all galaxies in region 4 as AGNs.

\subsection{Mid-infrared Luminosities of BCGs}

We convert the observed $24 \mu \mathrm{m}$ fluxes to inferred total IR luminosities following the models of Chary \& Elbaz (2001). This assumes that all of the $24 \mu \mathrm{m}$ flux is emitted by the BCG 
Table 2

Infrared-detected BCGs

\begin{tabular}{|c|c|c|c|c|c|c|}
\hline R.A. & Decl. & Redshift $^{\mathrm{a}}$ & $S_{24 \mu \mathrm{m}}(\mathrm{mJy})$ & $L_{\mathrm{IR}}\left(10^{11} L_{\odot}\right)^{\mathrm{b}}$ & $\operatorname{SFR}\left(M_{\odot} \mathrm{yr}^{-1}\right)$ & Stellar Mass $\left(10^{11} M_{\odot}\right)$ \\
\hline $10: 47: 22.8$ & $57: 00: 51$ & 0.238 & $1500.4 \pm 15.5$ & $0.71 \pm 0.36$ & $12 \pm 6$ & 0.9 \\
\hline $10: 33: 34.0$ & $58: 14: 35$ & 0.430 & $17900.7 \pm 28.6$ & $16.1 \pm 1.2$ & $281 \pm 218$ & 1.0 \\
\hline $10: 49: 22.3$ & $56: 56: 43$ & 0.244 & $1429.9 \pm 16.7$ & $1.9 \pm 1.3$ & $33 \pm 24$ & 2.4 \\
\hline $10: 48: 50.3$ & $56: 09: 08$ & 0.468 & $187.0 \pm 17.3$ & $0.34 \pm 0.24$ & $6 \pm 4$ & 1.2 \\
\hline $10: 42: 24.6$ & $57: 59: 49$ & 0.387 & $627.3 \pm 16.5$ & $1.1 \pm 0.8$ & $19 \pm 13$ & 0.6 \\
\hline 10:39:30.1 & $57: 14: 35$ & 0.425 & $386.9 \pm 18.6$ & $0.94 \pm 0.58$ & $17 \pm 10$ & 0.6 \\
\hline $10: 50: 56.6$ & $57: 07: 20$ & 0.475 & $221.4 \pm 12.2$ & $0.66 \pm 0.40$ & $12 \pm 7$ & 0.6 \\
\hline $10: 37: 38.9$ & $58: 45: 29$ & 0.688 & $329.0 \pm 16.8$ & $2.2 \pm 0.7$ & $39 \pm 12$ & 1.8 \\
\hline $10: 51: 57.3$ & $56: 59: 05$ & 0.700 & $638.7 \pm 12.8$ & $4.4 \pm 1.4$ & $77 \pm 24$ & 1.0 \\
\hline $10: 44: 48.9$ & $59: 12: 16$ & 0.737 & $239.0 \pm 12.7$ & $1.9 \pm 0.5$ & $32 \pm 8$ & 1.0 \\
\hline 10:39:46.1 & $58: 54: 17$ & 0.750 & $1380.2 \pm 17.1$ & $11.0 \pm 0.4$ & $192 \pm 72$ & 2.8 \\
\hline $10: 42: 28.6$ & $57: 51: 24$ & 0.778 & $464.0 \pm 17.6$ & $4.1 \pm 1.1$ & $71 \pm 19$ & 1.0 \\
\hline $10: 37: 25.8$ & $59: 02: 12$ & 0.813 & $248.5 \pm 13.5$ & $2.3 \pm 0.6$ & $39 \pm 10$ & 1.4 \\
\hline $10: 42: 36.0$ & $58: 18: 16$ & 0.825 & $163.3 \pm 14.8$ & $1.5 \pm 0.4$ & $26 \pm 7$ & 1.0 \\
\hline 10:40:05.0 & $59: 16: 26$ & 0.825 & $266.7 \pm 16.9$ & $2.5 \pm 0.7$ & $43 \pm 12$ & 1.4 \\
\hline $10: 35: 36.5$ & $57: 50: 35$ & 0.875 & $175.4 \pm 15.8$ & $1.7 \pm 0.6$ & $29 \pm 10$ & 1.1 \\
\hline $10: 58: 24.5$ & $57: 27: 26$ & 0.888 & $234.1 \pm 15.5$ & $2.3 \pm 0.8$ & $41 \pm 14$ & 1.3 \\
\hline $10: 46: 43.4$ & $58: 21: 20$ & 0.900 & $411.5 \pm 13.9$ & $4.5 \pm 1.5$ & $78 \pm 26$ & 2.4 \\
\hline $10: 48: 17.1$ & $55: 52: 18$ & 0.900 & $785.9 \pm 16.5$ & $9.1 \pm 3.5$ & $159 \pm 62$ & 1.0 \\
\hline $10: 44: 05.0$ & $58: 59: 04$ & 0.925 & $514.7 \pm 11.9$ & $6.2 \pm 2.2$ & $109 \pm 39$ & 1.4 \\
\hline $10: 51: 55.3$ & $57: 01: 09$ & 0.937 & $269.5 \pm 11.2$ & $3.2 \pm 1.2$ & $56 \pm 20$ & 0.6 \\
\hline $10: 55: 56.3$ & $57: 10: 37$ & 0.950 & $560.9 \pm 17.5$ & $7.4 \pm 2.9$ & $130 \pm 50$ & 0.8 \\
\hline $10: 34: 05.4$ & $58: 11: 31$ & 0.963 & $523.4 \pm 18.1$ & $7.2 \pm 2.9$ & $126 \pm 51$ & 1.9 \\
\hline $10: 52: 22.7$ & $56: 55: 44$ & 0.963 & $525.8 \pm 11.6$ & $7.3 \pm 2.8$ & $127 \pm 49$ & 1.1 \\
\hline $10: 46: 25.0$ & $57: 04: 29$ & 1.000 & $189.6 \pm 15.2$ & $2.7 \pm 1.3$ & $47 \pm 22$ & 1.7 \\
\hline $10: 47: 05.7$ & $58: 05: 42$ & 1.013 & $2551.0 \pm 19.7$ & $54.1 \pm 20.3$ & $947 \pm 356$ & 2.6 \\
\hline $10: 52: 06.0$ & $56: 56: 30$ & 1.038 & $485.5 \pm 11.8$ & $8.8 \pm 3.8$ & $153 \pm 66$ & 1.7 \\
\hline $10: 47: 49.7$ & $57: 43: 37$ & 1.029 & $388.9 \pm 14.1$ & $6.9 \pm 2.8$ & $120 \pm 50$ & 1.0 \\
\hline $10: 47: 38.8$ & $57: 52: 29$ & 1.075 & $1281.6 \pm 18.0$ & $32.5 \pm 12.2$ & $570 \pm 213$ & 2.0 \\
\hline $10: 59: 55.7$ & $57: 45: 56$ & 1.075 & $431.0 \pm 17.9$ & $8.9 \pm 3.9$ & $155 \pm 69$ & 1.2 \\
\hline $10: 52: 30.0$ & $58: 19: 43$ & 1.125 & $357.4 \pm 15.5$ & $8.6 \pm 3.7$ & $150 \pm 65$ & 1.6 \\
\hline $10: 46: 26.4$ & $57: 32: 30$ & 1.125 & $926.6 \pm 16.0$ & $27.0 \pm 10.1$ & $472 \pm 177$ & 1.5 \\
\hline 10:59:14.9 & $57: 32: 40$ & 1.200 & $226.4 \pm 14.4$ & $6.6 \pm 3.1$ & $116 \pm 54$ & 1.4 \\
\hline $10: 52: 08.4$ & $57: 02: 38$ & 1.250 & $377.2 \pm 11.1$ & $15.6 \pm 0.7$ & $272 \pm 123$ & 2.2 \\
\hline 10:50:07.2 & $57: 16: 51$ & 1.600 & $662.7 \pm 12.3$ & $82.2 \pm 24.3$ & $1438 \pm 426$ & 3.6 \\
\hline $10: 44: 59.5$ & $57: 52: 07$ & 1.650 & $281.1 \pm 17.0$ & $34.2 \pm 9.0$ & $599 \pm 152$ & 5.3 \\
\hline $10: 49: 22.6$ & $56: 40: 33$ & 1.70 & $606.1 \pm 18.0$ & $104 \pm 29$ & $1819 \pm 511$ & 4.5 \\
\hline $02: 19: 41.8$ & $-04: 00: 33.3$ & 0.141 & $358.1 \pm 18.4$ & $0.12 \pm 0.06$ & $2 \pm 1$ & 0.5 \\
\hline $02: 25: 24.9$ & $-03: 47: 35.4$ & 0.250 & $263.8 \pm 16.3$ & $0.18 \pm 0.09$ & $3 \pm 2$ & 0.3 \\
\hline $02: 24: 26.9$ & $-05: 36: 32.2$ & 0.262 & $1814.7 \pm 17.8$ & $1.1 \pm 0.5$ & $19 \pm 9$ & 0.7 \\
\hline $02: 24: 33.1$ & $-04: 53: 56.1$ & 0.337 & $262.34 \pm 19.7$ & $0.34 \pm 0.29$ & $6 \pm 5$ & 0.5 \\
\hline 02:19:10.5 & $-03: 43: 34.4$ & 0.763 & $2684.4 \pm 18.8$ & $18.8 \pm 7.6$ & $329 \pm 132$ & 1.6 \\
\hline $02: 20: 13.3$ & $-06: 00: 54.9$ & 0.688 & $339.5 \pm 20.9$ & $2.3 \pm 0.8$ & $40 \pm 13$ & 1.1 \\
\hline $02: 20: 27.8$ & $-05: 47: 24.9$ & 0.762 & $236.0 \pm 16.4$ & $1.9 \pm 0.5$ & $34 \pm 9$ & 1.2 \\
\hline $02: 22: 13.0$ & $-04: 21: 58.4$ & 0.788 & $318.1 \pm 20.2$ & $2.8 \pm 0.8$ & $48 \pm 13$ & 0.9 \\
\hline $02: 24: 24.0$ & $-02: 58: 02.7$ & 0.788 & $216.3 \pm 19.6$ & $1.9 \pm 0.5$ & $33 \pm 9$ & 0.9 \\
\hline 02:19:29.0 & $-04: 07: 00.2$ & 0.813 & $1086.4 \pm 20.4$ & $10.3 \pm 3.8$ & $180 \pm 66$ & 1.6 \\
\hline 02:18:34.4 & $-05: 00: 43.6$ & 0.651 & $368.8 \pm 19.0$ & $3.5 \pm 1.0$ & $61 \pm 17$ & 1.7 \\
\hline $02: 15: 43.9$ & $-04: 24: 53.5$ & 0.850 & $694.6 \pm 18.2$ & 7. \pm 2.0 & $122 \pm 37$ & 2.7 \\
\hline $02: 24: 29.2$ & $-04: 10: 13.1$ & 0.900 & $1082.9 \pm 19.8$ & $12.4 \pm 5.4$ & $235 \pm 95$ & 1.3 \\
\hline $02: 25: 06.9$ & $-04: 47: 18.4$ & 0.925 & $276.0 \pm 19.4$ & $3.2 \pm 1.2$ & $55 \pm 21$ & 1.8 \\
\hline 02:02:08.5 & $-03: 41: 26.8$ & 0.925 & $345.9 \pm 19.3$ & $4.0 \pm 1.5$ & $70 \pm 27$ & 1.0 \\
\hline $02: 14: 38.2$ & $-03: 37: 38.3$ & 0.931 & $2241.6 \pm 22.2$ & $35.1 \pm 12.8$ & $614 \pm 224$ & 2.7 \\
\hline 02:23:05.8 & $-04: 13: 35.5$ & 1.048 & $240.1 \pm 18.2$ & $3.1 \pm 1.3$ & $53 \pm 23$ & 0.9 \\
\hline $02: 20: 54.8$ & $-03: 32: 57.5$ & 0.994 & $1137.5 \pm 21.7$ & $20.9 \pm 8.8$ & $356 \pm 153$ & 3.3 \\
\hline $02: 22: 36.5$ & $-03: 50: 30.3$ & 0.822 & $1039.2 \pm 19.7$ & $19.3 \pm 8.4$ & $338 \pm 146$ & 2.0 \\
\hline $02: 16: 38.2$ & $-03: 28: 44.6$ & 1.00 & $698.5 \pm 21.1$ & $11.3 \pm 5.5$ & $198 \pm 95$ & 2.0 \\
\hline $02: 22: 54.3$ & $-04: 14: 11.9$ & 1.00 & $362.3 \pm 21.3$ & $5.5 \pm 2.4$ & $96 \pm 42$ & 1.0 \\
\hline 02:16:03.2 & $-03: 33: 57.9$ & 1.05 & $352.5 \pm 16.4$ & $6.5 \pm 2.7$ & $113 \pm 47$ & 1.8 \\
\hline 02:18:05.2 & $-05: 00: 10.5$ & 1.095 & $247.1 \pm 15.9$ & $4.8 \pm 2.1$ & $83 \pm 36$ & 2.2 \\
\hline $02: 16: 52.3$ & $-03: 37: 56.7$ & 1.350 & $508.8 \pm 20.0$ & $32.0 \pm 11.7$ & $559 \pm 204$ & 2.3 \\
\hline $02: 26: 15.6$ & $-04: 56: 28.0$ & 1.375 & $182.9 \pm 19.7$ & $10.3 \pm 4.9$ & $180 \pm 87$ & 2.3 \\
\hline $02: 21: 43.8$ & $-03: 21: 57.0$ & 1.375 & $1023.9 \pm 21.0$ & $72.2 \pm 27.1$ & $1263 \pm 474$ & 3.8 \\
\hline $02: 27: 30.5$ & $-04: 32: 03.4$ & 1.450 & $435.9 \pm 18.2$ & $35.8 \pm 11.1$ & $628 \pm 195$ & 1.8 \\
\hline
\end{tabular}


Table 2

(Continued)

\begin{tabular}{|c|c|c|c|c|c|c|}
\hline R.A. & Decl. & Redshift $^{\mathrm{a}}$ & $S_{24 \mu \mathrm{m}}(\mathrm{mJy})$ & $L_{\mathrm{IR}}\left(10^{11} L_{\odot}\right)^{\mathrm{b}}$ & $\operatorname{SFR}\left(M_{\odot} \mathrm{yr}^{-1}\right)$ & Stellar Mass $\left(10^{11} M_{\odot}\right)$ \\
\hline $02: 18: 45.2$ & $-05: 42: 56.8$ & 1.45 & $313.3 \pm 19.6$ & $24.8 \pm 8.3$ & $434 \pm 145$ & 2.0 \\
\hline $16: 05: 44.6$ & $54: 57: 16.2$ & 0.188 & $378.6 \pm 16.8$ & $0.01 \pm 0.06$ & $2 \pm 1$ & 0.7 \\
\hline $16: 06: 27.9$ & $54: 56: 14.7$ & 0.188 & $231.0 \pm 12.9$ & $0.08 \pm 0.04$ & $1 \pm 0.7$ & 0.3 \\
\hline $16: 13: 32.9$ & $56: 17: 47.5$ & 0.212 & $683.5 \pm 17.4$ & $0.27 \pm 0.13$ & $5 \pm 2$ & 0.8 \\
\hline $16: 18: 38.3$ & $55: 17: 13.7$ & 0.250 & $107.5 \pm 13.2$ & $0.08 \pm 0.04$ & $1 \pm 1$ & 0.9 \\
\hline $16: 14: 51.5$ & 54: $02: 22.0$ & 0.275 & $1042.6 \pm 16.7$ & $0.72 \pm 0.36$ & $13 \pm 6$ & 0.5 \\
\hline $16: 19: 14.8$ & $55: 13: 40.8$ & 0.313 & $224.6 \pm 16.4$ & $0.21 \pm 0.21$ & $5 \pm 4$ & 0.7 \\
\hline $16: 10: 11.2$ & $53: 46: 24.5$ & 0.362 & $290.0 \pm 15.9$ & $0.44 \pm 0.37$ & $8 \pm 7$ & 0.9 \\
\hline $16: 13: 17.5$ & $56: 01: 19.3$ & 0.387 & $2571.9 \pm 18.8$ & $3.9 \pm 2.8$ & $68 \pm 49$ & 1.3 \\
\hline $16: 00: 37.2$ & $55: 27: 26.9$ & 0.465 & $163.5 \pm 16.2$ & $0.33 \pm 0.23$ & $6 \pm 4$ & 1.4 \\
\hline $16: 08: 57.2$ & $56: 00: 18.6$ & 0.688 & $524.1 \pm 17.0$ & $3.5 \pm 1.1$ & $61 \pm 20$ & 7.2 \\
\hline $16: 02: 32.3$ & $54: 56: 59.6$ & 0.700 & $150.0 \pm 14.5$ & $1.1 \pm 0.3$ & $18 \pm 6$ & 0.8 \\
\hline $16: 18: 40.1$ & $54: 50: 48.0$ & 0.700 & $759.9 \pm 15.1$ & $5.2 \pm 1.7$ & $91 \pm 29$ & 1.2 \\
\hline $16: 06: 28.8$ & $55: 33: 07.7$ & 0.725 & $165.3 \pm 16.1$ & $1.2 \pm 0.3$ & $22 \pm 6$ & 0.9 \\
\hline $16: 18: 26.4$ & $54: 58: 28.7$ & 0.737 & $278.2 \pm 13.0$ & $2.1 \pm 0.6$ & $38 \pm 10$ & 1.3 \\
\hline $16: 11: 01.3$ & $54: 17: 05.3$ & 0.750 & $695.7 \pm 15.7$ & $5.5 \pm 1.6$ & $97 \pm 28$ & 1.8 \\
\hline $16: 03: 04.5$ & $54: 57: 24.3$ & 0.775 & $607.1 \pm 15.9$ & $5.2 \pm 1.4$ & $90 \pm 25$ & 1.2 \\
\hline $16: 09: 29.4$ & $54: 29: 40.6$ & 0.788 & $1764.0 \pm 16.7$ & $17.3 \pm 6.0$ & $304 \pm 106$ & 0.8 \\
\hline $16: 17: 29.9$ & $55: 58: 18.2$ & 0.788 & $207.1 \pm 16.2$ & $1.8 \pm 0.5$ & $31 \pm 8$ & 1.0 \\
\hline $16: 13: 05.7$ & $55: 59: 50.0$ & 0.825 & $169.9 \pm 13.8$ & $1.5 \pm 0.4$ & $27 \pm 7$ & 1.4 \\
\hline 16:07:16.8 & $55: 32: 59.8$ & 0.825 & $600.2 \pm 13.8$ & $5.7 \pm 1.6$ & $100 \pm 28$ & 1.1 \\
\hline $16: 08: 25.6$ & $54: 45: 08.9$ & 0.906 & $610.6 \pm 13.4$ & $5.8 \pm 1.6$ & $102 \pm 29$ & 0.8 \\
\hline $16: 19: 19.7$ & $55: 36: 45.7$ & 0.862 & $299.8 \pm 13.9$ & $2.9 \pm 0.9$ & $51 \pm 15$ & 0.6 \\
\hline $16: 02: 50.7$ & $54: 54: 52.2$ & 0.862 & $337.9 \pm 14.7$ & $3.3 \pm 1.0$ & $58 \pm 17$ & 0.9 \\
\hline $16: 12: 10.4$ & $56: 07: 53.6$ & 0.888 & $483.1 \pm 13.9$ & $5.2 \pm 1.7$ & $90 \pm 29$ & 0.8 \\
\hline $16: 11: 12.7$ & $55: 08: 23.6$ & 0.907 & $3065.4 \pm 19.9$ & $43.7 \pm 15.8$ & $766 \pm 275$ & 1.8 \\
\hline 16:08:07.8 & $54: 19: 43.1$ & 0.900 & $765.5 \pm 17.5$ & $8.8 \pm 3.3$ & $154 \pm 57$ & 1.4 \\
\hline $16: 18: 18.0$ & $54: 35: 38.9$ & 0.944 & $671.7 \pm 17.0$ & $8.8 \pm 3.7$ & $155 \pm 66$ & 1.8 \\
\hline $16: 22: 42.1$ & $54: 50: 55.0$ & 1.000 & $390.7 \pm 19.7$ & $6.0 \pm 2.6$ & $105 \pm 45$ & 1.1 \\
\hline $16: 02: 43.5$ & $54: 49: 09.7$ & 1.013 & $194.3 \pm 17.4$ & $2.9 \pm 1.4$ & $51 \pm 24$ & 0.8 \\
\hline $16: 13: 01.7$ & $54: 46: 10.0$ & 1.092 & $1220.8 \pm 14.5$ & $24.2 \pm 9.5$ & $423 \pm 167$ & 1.8 \\
\hline $16: 08: 27.1$ & $54: 36: 47.3$ & 1.125 & $345.3 \pm 14.0$ & $8.3 \pm 3.3$ & $145 \pm 58$ & 1.2 \\
\hline $16: 07: 25.7$ & $54: 40: 41.2$ & 1.125 & $250.6 \pm 15.0$ & $5.8 \pm 2.5$ & $101 \pm 43$ & 0.7 \\
\hline $16: 11: 15.8$ & $54: 15: 11.4$ & 1.125 & $365.7 \pm 15.6$ & $8.8 \pm 3.9$ & $153 \pm 69$ & 1.3 \\
\hline $16: 05: 55.2$ & $55: 31: 47.2$ & 1.350 & $326.0 \pm 14.2$ & $19.5 \pm 8.0$ & $342 \pm 138$ & 2.6 \\
\hline $16: 06: 52.7$ & $55: 39: 36.6$ & 1.650 & $241.9 \pm 16.5$ & $28.3 \pm 7.3$ & $496 \pm 128$ & 4.6 \\
\hline $16: 40: 33.2$ & $41: 23: 11.7$ & 0.662 & $220.6 \pm 16.2$ & $1.4 \pm 0.5$ & $25 \pm 8$ & 1.6 \\
\hline $16: 34: 35.3$ & $41: 36: 14.6$ & 0.781 & $584.5 \pm 15.1$ & $4.2 \pm 1.3$ & $73 \pm 22$ & 1.3 \\
\hline $16: 38: 22.9$ & $41: 53: 19.1$ & 0.862 & $772.1 \pm 16.4$ & $8.0 \pm 2.5$ & $141 \pm 44$ & 1.3 \\
\hline 16: $37: 35.1$ & $40: 17: 18.1$ & 0.912 & $217.1 \pm 16.3$ & $2.3 \pm 0.9$ & $40 \pm 16$ & 2.9 \\
\hline $16: 41: 52.4$ & 41:34:01.5 & 0.925 & $297.8 \pm 17.8$ & $3.4 \pm 1.3$ & $60 \pm 23$ & 0.7 \\
\hline $16: 37: 49.5$ & $42: 04: 55.3$ & 0.931 & $2767.4 \pm 18.5$ & $43.7 \pm 16.1$ & $765 \pm 281$ & 3.7 \\
\hline $16: 37: 01.1$ & $41: 31: 02.4$ & 0.944 & $747.8 \pm 17.1$ & $10.0 \pm 4.5$ & $175 \pm 78$ & 1.3 \\
\hline $16: 37: 46.5$ & $40: 37: 32.3$ & 0.944 & $507.1 \pm 17.4$ & $6.5 \pm 2.5$ & $114 \pm 44$ & 2.3 \\
\hline $16: 31: 06.1$ & $40: 50: 31.3$ & 0.956 & $340.8 \pm 17.8$ & $4.3 \pm 1.8$ & $76 \pm 31$ & 1.1 \\
\hline 16:38:07.1 & $41: 57: 34.2$ & 1.075 & $362.9 \pm 16.4$ & $7.3 \pm 3.0$ & $128 \pm 53$ & 0.8 \\
\hline $16: 40: 22.3$ & 42:03:14.4 & 1.125 & $295.8 \pm 13.3$ & $7.0 \pm 2.9$ & $122 \pm 50$ & 1.2 \\
\hline $16: 37: 27.0$ & 41:57:00.6 & 1.125 & $334.7 \pm 13.3$ & $8.0 \pm 3.2$ & $139 \pm 57$ & 1.3 \\
\hline $16: 41: 40.3$ & 41:13:18.4 & 1.125 & $283.4 \pm 15.0$ & $6.4 \pm 2.8$ & $116 \pm 49$ & 1.3 \\
\hline $16: 39: 12.2$ & $40: 57: 25.4$ & 1.275 & $269.8 \pm 16.1$ & $10.9 \pm 5.7$ & $190 \pm 100$ & 1.7 \\
\hline $16: 37: 59.2$ & $42: 03: 56.5$ & 1.575 & $175.0 \pm 12.9$ & $17.0 \pm 5.0$ & $293 \pm 87$ & 1.7 \\
\hline $16: 37: 37.9$ & $41: 17: 28.6$ & 1.775 & $339.4 \pm 17.9$ & $52.9 \pm 14.3$ & $925 \pm 249$ & 2.2 \\
\hline
\end{tabular}

Notes.

${ }^{\text {a }}$ Bold denotes a spectroscopic redshift, non-bold corresponds to the red-sequence-estimated redshift.

${ }^{\mathrm{b}}$ LIRs estimated using the $24 \mu \mathrm{m}$ flux and Chary \& Elbaz (2001) methodology.

itself (that is, no gravitational lensing) and is entirely due to star formation with no AGN contamination. We therefore highlight, when relevant, those galaxies which may contain AGNs, as identified in the previous section (Donley et al. 2012).
In Figure 5, we show the infrared luminosity of the $24 \mu \mathrm{m}$ detected BCGs with redshift, and we list the values in Table 2. We include for reference the Herschel-detected BCGs of Rawle et al. (2012) and the extreme star-forming BCG in the Phoenix 


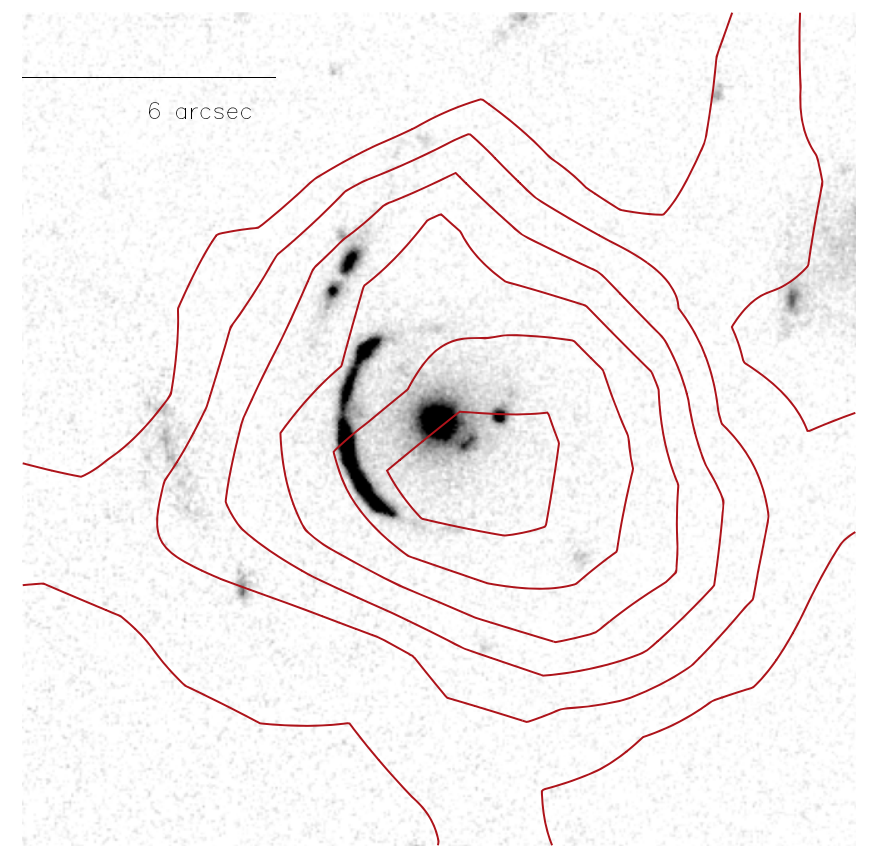

Figure 3. HST-ACS-606W image one of the BCGs in this sample (from the CANDELS UDS public data) that is clearly lensing a background galaxy. In red we show the MIPS flux contours starting at $0.05 \mathrm{MJy} \mathrm{sr}^{-1}$ and increasing in 0.05 steps. Given the beam size of MIPS ( $6^{\prime \prime}$ FWHM shown), the source of the $24 \mu \mathrm{m}$ emission is ambiguous.

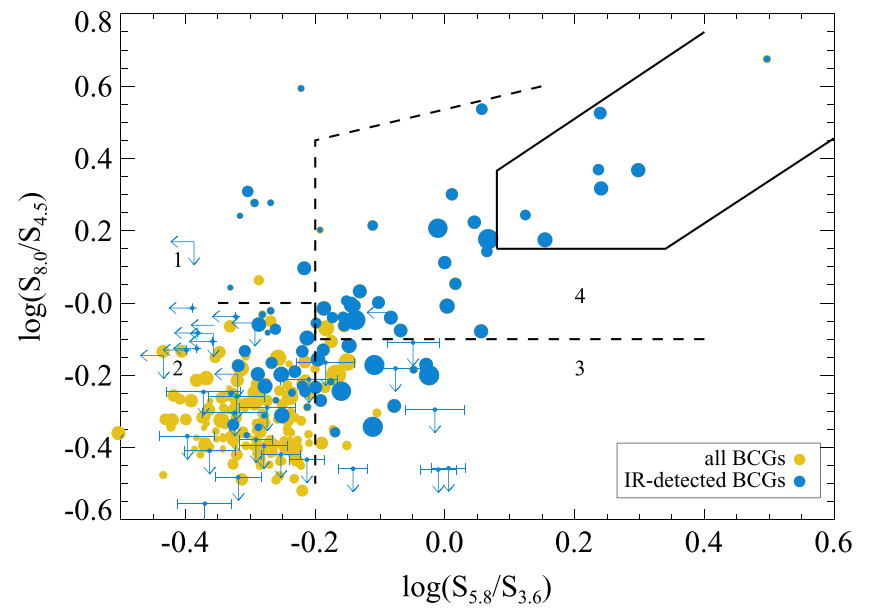

Figure 4. IRAC color-color plot for the SpARCS BCGs. Blue points correspond to $24 \mu \mathrm{m}$ detected BCGs, and orange to $24 \mu \mathrm{m}$ non-detected BCGs. The increasing sizes of the points correspond to increasing redshift. We also show $24 \mu \mathrm{m}$ detected BCGs that have limits on their 5.8 or $8.0 \mu \mathrm{m}$ flux, but for clarity only include the infrared-faint BCGs that have detections in all four IRAC channels. Overlaid are the Sajina et al. (2005) SED template sections (dotted divisions; 1: low $-z$ PAH; 2 and 3: mid-/high- $z$ PAH and stellar continuum; 4: high- $z$ PAH and all- $z$ AGN) and the more recent Donley et al. (2012) AGN wedge (solid line).

cluster reported by McDonald et al. (2012). The Phoenix BCG is an outlier at $z=0.6$, whereas the IR luminosities of the Rawle et al. sample are comparable to ours, with a few exceptions. Note, however, that the Rawle et al. clusters are $\mathrm{X}$-ray selected and located at redshift $z<1.0$, whereas our sample is effectively stellar-mass selected to higher redshift, and therefore a direct comparison of the two samples is not straightforward.

There is a consistent rise in Figure 5 in the inferred infrared luminosities of BCGs with similar cluster richness out to $z \sim 2$.

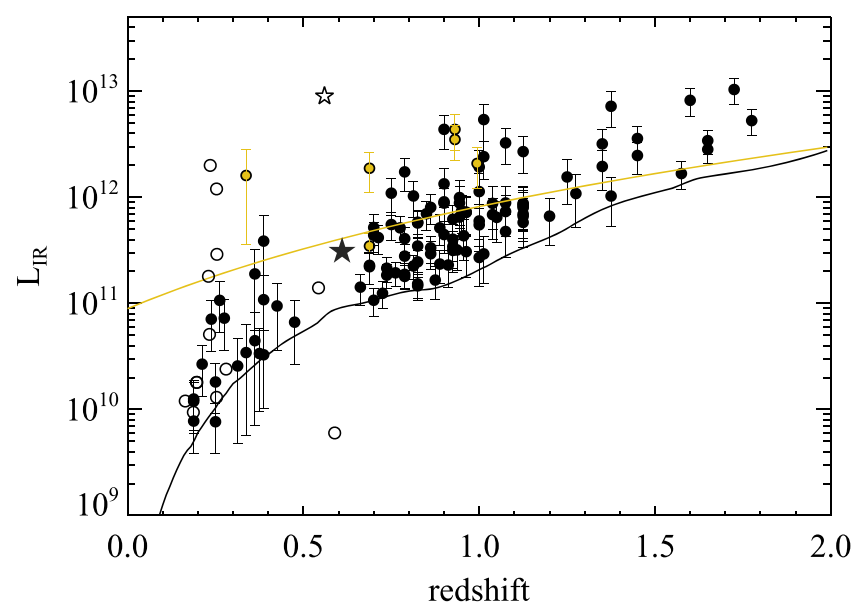

Figure 5. $24 \mu \mathrm{m}$ inferred infrared luminosity (assuming the models of Chary \& Elbaz 2001). Solid points refer to the SpARCS cluster sample (this work), with the orange color denoting possible AGNs, as identified by the Donley et al. (2012) region in Figure 4. The error bars include the $24 \mu \mathrm{m}$ flux uncertainties and a \pm 0.1 redshift scatter. The open circles correspond to the Herscheldetected BCGs of Rawle et al. (2012), although we use their $24 \mu \mathrm{m}$ estimated $L_{\mathrm{IR}}$ for consistency. The open star denotes the extreme star-forming BCG reported by McDonald et al. (2012), discovered in the SPT survey. The solid star corresponds to the BCG with a strongly lensed arc (Section 3.3.2); here, we assume the source of the $24 \mu \mathrm{m}$ flux is the BCG itself. Note that this object is not actually part of our study as it has $N_{\text {gal }}<12$, but it is shown for reference. The orange solid line shows the luminosity depth discussed in Section 4.2, which is tied to an evolving IR luminosity function, and the solid black line shows the approximate luminosity depth of the SWIRE $24 \mu \mathrm{m}$ survey.

Given that the infrared luminosity function of field galaxies evolves toward higher luminosities and densities over this redshift range, this evolution may simply be a reflection of this general trend. In Figure 5, we show an evolving infrared luminosity limit following the $L_{\mathrm{IR}} \sim(1+z)^{3.2}$ relation of Le Floc'h et al. (2005) and scaled to our highest luminosity depth $\left(\sim L_{\mathrm{IR}}=1 \times 10^{12} L_{\odot}\right.$ at $\left.z=1.8\right)$. This defines the luminosity limit as the same fraction of an evolving $L_{\mathrm{IR}}^{*}$, at every redshift, where, for reference, $L_{\mathrm{IR}}^{*} \sim 1.6 \times 10^{10} L_{\odot}$ at $z \sim 1$.

There is still an increase in the absolute number of objects above this line; however, this should not be interpreted as a luminosity evolution. The absolute numbers of BCGs at a given luminosity will depend on the volume probed (which increases by $\sim 3$ from $0<z<1$ to $1<z<2$ ), as well as the cluster number density and selection function.

In Figure 6, we show the fraction of BCGs that are detected above a given luminosity limit that is more likely to trace a real change in clusters with time. In the top panel of Figure 6, we show the fraction of all BCGs detected at different luminosity/ flux limits. We include the fraction of BCGs detected at any flux above the depth of SWIRE $(\sim 100 \mu \mathrm{Jy})$; above $L_{\mathrm{IR}}>10^{12}$ $L_{\odot}$, and above the field-related evolving infrared limit shown in Figure 5. In all cases, the fraction of IR-bright BCGs increases significantly beyond $z \sim 1$ for similar richness clusters. In particular, the field-corrected evolution increases from $\sim 5 \%$ to $\sim 30 \%$ from the lowest- to highest-redshift bins. This plot includes all IR-detected systems, even those with possible AGN contamination. In the lower panel, we show the trend after removing all galaxies within the Sajina et al. (2005) region 4, which as explained in Section 4, we take to be the highest level of AGN contamination. Using the Donley et al. region provides a much lower contamination rate. While the evolution is reduced by roughly a factor of two (for the Sajina et al. 

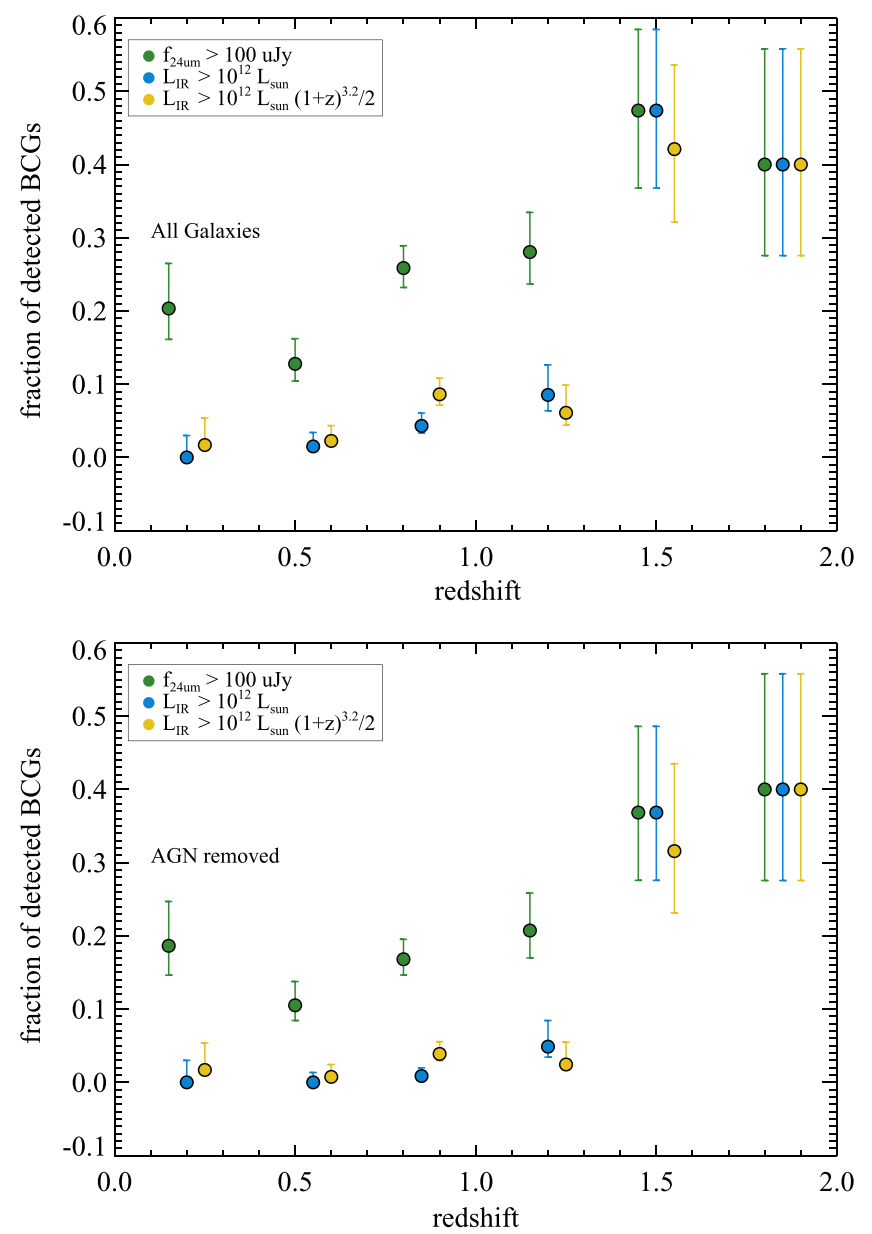

Figure 6. Top: the fraction of $24 \mu \mathrm{m}$ detected BCGs in different redshift bins. Blue points denote BCGs with inferred infrared luminosities of $>L_{\mathrm{IR}} 10^{12} L_{\odot}$ and orange points denote the fraction of BCGs above the evolving luminosity cut shown by the orange line in Figure 5. Also shown (green) are the fractions of BCGs detected at any $24 \mu \mathrm{m}$ flux above the detection limit of the SWIRE survey. Bottom: same as the top panel, but with possible AGN contaminated galaxies removed from the analysis. For this plot, we define as AGNs those galaxies lying in region 4 of Figure 4.

contamination rate), it remains significant, in part because the AGNs are scattered throughout most of the redshift bins.

We note, finally, a systematic effect that may be present in this analysis. Several studies have shown that the Chary \& Elbaz (2001) methodology overestimates the infrared luminosity above a redshift of $z \sim 1.5$ (Murphy et al. 2009; Rodighiero et al. 2010; Nordon et al. 2012) by roughly a factor of five due to large PAH equivalent widths at these redshifts. This redshift transition is the approximate location where we see the largest change in the fraction of $24 \mu \mathrm{m}$ detected BCGs. It may be that due to this effect, we are sensitive to a lower luminosity limit beyond $z \sim 1.5$, which would in turn lead to a larger fraction of detected galaxies. If we applied a downward correction to the $L_{\mathrm{IR}}$ in Figure 5, this would indeed flatten the high-redshift tail and bring those estimations more in line with the measurements below $z \sim 1.5$, but it would not change our results qualitatively as these systems would still remain at $L_{\mathrm{IR}}>10^{12} L_{\odot}$. Moreover, the infrared luminosity of the most luminous object in our sample, SpARCS1049+56 at $z=1.7$, has been studied in detail in Webb et al. (2015), where we determine its luminosity using six infrared measurements and two limits. We determine the $L_{\mathrm{IR}}$ to be $6.6 \pm 0.9 \times 10^{12} L_{\odot}$,

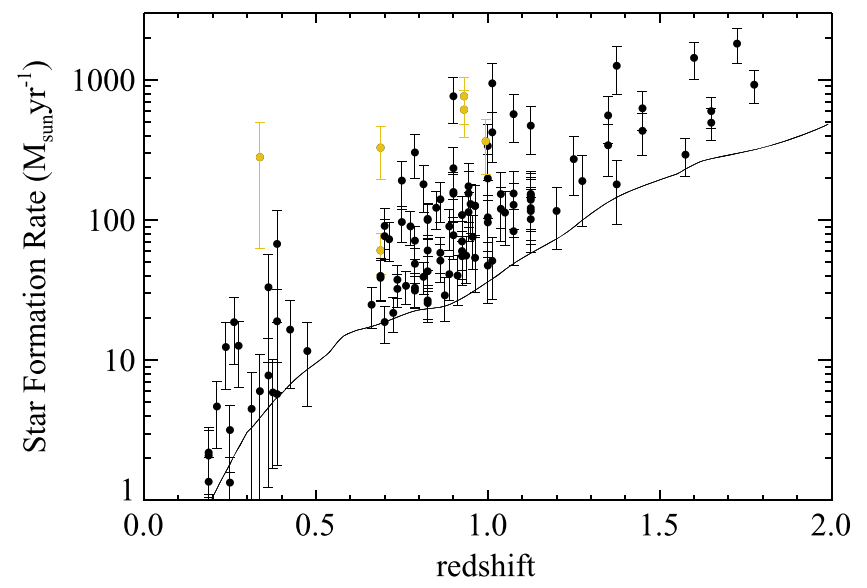

Figure 7. Inferred star formation rates of the BCGs with redshift. The orange points correspond to those with IRAC colors consistent with AGNs following the Donley et al. (2012) criteria. The solid line shows the approximate depth of the SWIRE $24 \mu \mathrm{m}$ imaging.

compared to an $L_{\mathrm{IR}}$ of $1.0 \pm 0.3 \times 10^{13} L_{\odot}$ determined from the $24 \mu \mathrm{m}$ measurement alone. These estimates are within $1 \sigma$ of each other indicating, at least for this particular object, there is no strong overestimate of the flux.

\subsection{SFRs of the BCGs}

We can scale the infrared luminosities determined in the previous section to SFRs following the relation of Kennicutt (1998). Based on Figure 4, we assume no AGN contribution to the infrared flux, which would systematically reduce the estimates. We show the SFRs for each BCG with redshift in Figure 7. Uncertainties include the photometric uncertainty in the MIPS $24 \mu \mathrm{m}$ flux and the redshift uncertainty of $\Delta z \pm 0.1$. A wide range in SFRs is seen from $\sim 1$ at low redshift to $\sim 1000 M_{\odot} \mathrm{yr}^{-1}$ for the highest-redshift BCGs.

Using stellar-mass measurements for the BCGs, we can further convert the SFRs to SFRs per unit stellar mass, or specific star formation rates (sSFRs). Given the uniformity of the BCG optical colors, we adopt a simple methodology to measure stellar mass. We use the observed $3.6 \mu \mathrm{m}$ flux to determine the rest-frame $K$-band luminosity, adopting an $11 \mathrm{Gyr}$ single stellar population from Bruzual \& Charlot (2003) to calculate the $K$-correction. We then take the average $K$-band mass-to-light ratio of a red galaxy $\left(M / L_{K}=1\right)$ from Bell et al. (2003), with a scatter of 0.1dex. To determine the sSFR, we further scale the masses by 1.65 to convert to a Salpeter IMF (Raue \& Meyer 2012).

Figure 8 shows the sSFRs of the individual BCGs with redshift. This figure also contains the evolution of the sSFR of the main sequence of star-forming galaxies (Elbaz et al. 2011; solid line), as well as the division between main-sequence and starburst systems as defined by Elbaz et al.

In Figure 9, we show the sSFR of the $24 \mu \mathrm{m}$ detected BCGs as a function of stellar mass. The size of the points increases with the redshift of the BCG so that the largest points correspond to at $z=1.8$ and the smallest points to $z=0.1$. Overlaid we show the location of the main sequence of starforming galaxies in the field for the three redshift regimes that encompass our sample: $z=0,1,2$ (Daddi et al. 2007; Elbaz et al. 2007), again corrected to a Salpeter IMF.

In both Figures 8 and 9, general agreement is seen between the sSFRs of the BCGs and the overall level seen in the field at 


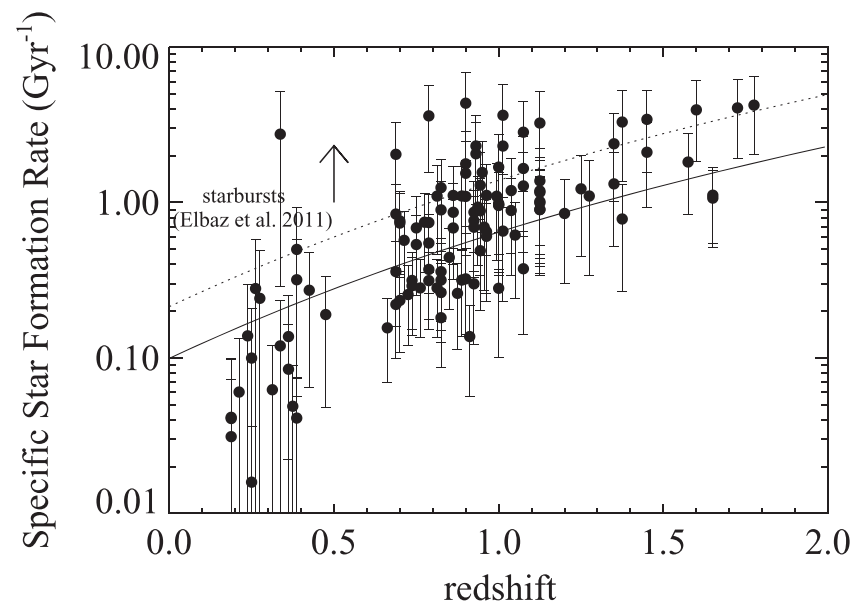

Figure 8. Specific star formation rate of the $24 \mu \mathrm{m}$ detected BCGs shown as a function of redshift. Overlaid (solid line) is the best-fit relation of Elbaz et al. (2011) for main-sequence star-forming galaxies. The dotted line shows the rough division between starburst galaxies and main-sequence galaxies, as classified by Elbaz et al. No AGN correction has been made to the SFRs.

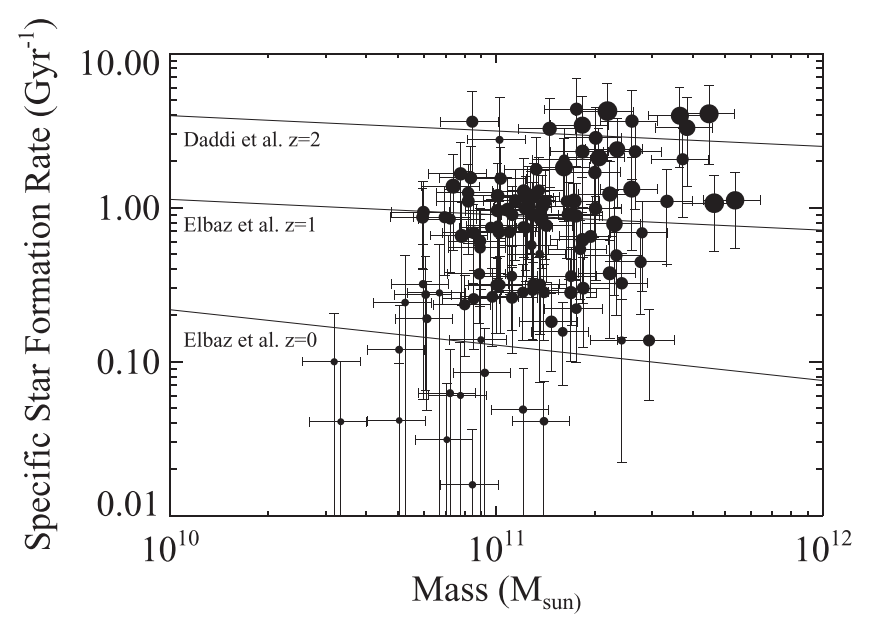

Figure 9. Specific star formation rate of the $24 \mu \mathrm{m}$ detected BCGs shown as a function of stellar mass. Overlaid are the relations for main-sequence starforming galaxies at the redshifts $z=0,1$ (Elbaz et al. 2007) and $z=2$ (Daddi et al. 2007) that encompass our sample. The size of the data point, for each $\mathrm{BCG}$, increases with increasing redshift from $z \sim 0.1$ to 1.8 .

a given redshift or mass. This indicates that although the star formation activity within the BCGs is quantitatively high, it is not in great excess of that seen for field galaxies, and indeed the BCGs may be classified as main-sequence star-forming systems.

\section{DISCUSSION}

\subsection{A Change in the Observed Activity in BCGs with Redshift}

These data show a measurable change in the IR properties of similarly selected BCGs from redshift $z \sim 0.3$ to $z \sim 1.8$. The IR colors indicate that the bulk of this evolution is due to an increase in the SFRs of BCGs to higher redshift and the remainder from dusty AGNs. This is qualitatively similar to the rapid increase of the global SFR density of the universe to $z \sim$ 1 , as well as the migration of $L_{\mathrm{IR}}^{\star}$ to higher luminosities over the same redshift range. Figure 6 suggests, however, that the BCGs may follow a steeper evolution than seen for the field. We cannot constrain the slope of this evolution because we are only sensitive to the most luminous galaxies at high redshifts; however, we do see a clear increase in the fraction of BCGs detected above an IR depth that is defined relative to the evolution of the field IR luminosity function (see Section 4.2). Note that this result is not driven by the increase in AGN contamination: it remains when all of the candidate AGNs are removed from the sample. This is evidence that the physics driving the increased activity in BCGs at higher redshift is either different than that driving the field evolution or is accelerated in the cores of galaxy clusters.

\subsection{The Stellar Mass Growth of BCGs}

Below $z \sim 1$, the SFRs of individual detected BCGs do not exceed $100 M_{\odot} \mathrm{yr}^{-1}$. If the detection rate of BCGs at this level $(\sim 10 \%)$ is reflective of the duty cycle of the starburst, then they must be short lived, or $\lesssim 1$ Gyr. At such rates, this star formation will contribute a relatively small amount of stellar mass to the overall system. There have been conflicting measurements of the actual stellar-mass growth rate of BCGs in this redshift range, but generally an increase between $\sim 0-2 \times$ is seen since $z=1$ (Lidman et al. 2012; Liu et al. 2012). This mass growth has been attributed by many to the accretion of established stellar populations through gas-poor major or minor galaxy mergers (Edwards \& Patton 2012; Lidman et al. 2013). The IR results seen here (below $z<1$ ) do not add enough stellar mass to contradict this scenario.

By $z \sim 1$, however, the star formation level has drastically increased to $\sim 500-2000 M_{\odot} \mathrm{yr}^{-1}$. Using similar arguments as above we reach similar timescale conclusions: if the duty cycle is $20 \%$ over the epoch studied, then the bursts must be limited to a few hundred million years. In this case, however, the greatly increased SFRs mean that even over this short timescale a BCG can easily double its stellar mass. At these levels, in situ star formation is an important, perhaps dominant, contributor to the mass growth of BCGs (Collins 2009; Stott et al. 2011; Lidman et al. 2013).

This general reasoning can also be illustrated in Figure 10, which is adapted from Lidman et al. (2012). Using NIRdetermined mass measurements of BCGs, Lidman et al. calculated the fraction of $z=0$ stellar mass in BCGs in place at a given redshift to $z \sim 1$. They find relatively good agreement with the buildup of mass due to dry mergers in the SAMS of De Lucia \& Blaizot (2007). These two studies are shown in Figure 10, but are plotted as the amount of mass still to be added to a BCG at a given redshift as a fraction of the final mass. We compare to this the amount of stellar mass added by the star formation seen here, again in terms of the final stellar mass at $z=0$, which is shown by the gray area.

To determine the gray area, we measure the average SFR for the entire BCG population in redshift bins, assigning an SFR of $0 M_{\odot} \mathrm{yr}^{-1}$ to the undetected BCGs. The chosen bins are $z=0.2-0.6,0.6-1.0,1.0-1.4,1.4-1.8$. We then integrate this star formation over the duration of the redshift bin to obtain the average amount of stellar mass added in that bin. This is then combined with the existing average stellar mass of the BCGs within the redshift bin to provide a total, final mass, treating each bin independently. This is complementary to our reasoning at the very beginning of this section where we took the high SFRs of the IR-detected BCGs and used the detected fraction to constrain the limits of integration (the duty cycle). Here, we do not constrain the timescale of the star formation-allowing the star formation to proceed at the same rate over the entire 


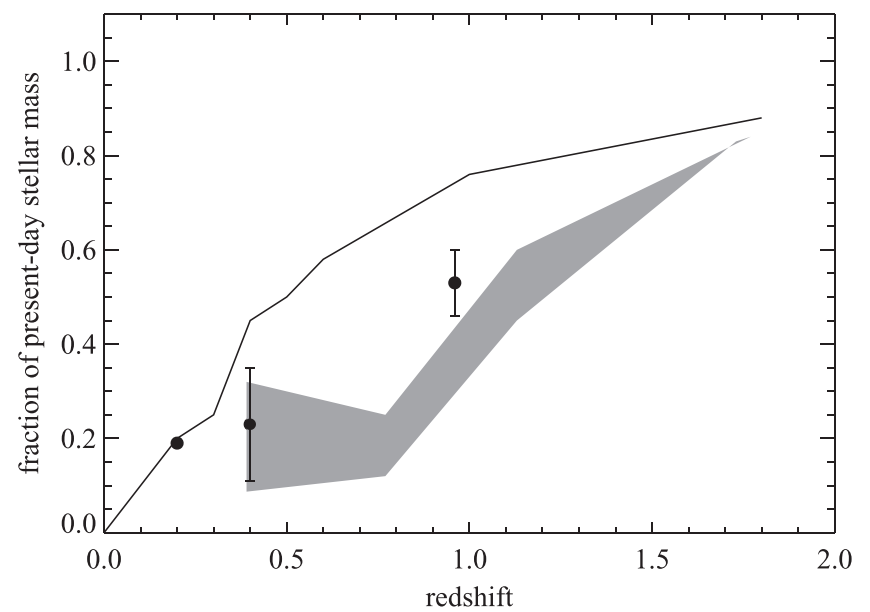

Figure 10. This plot, which is adapted from Lidman et al. (2012), shows the amount of stellar mass that is added to BCGs with time, normalized to their present-day $(z=0)$ stellar mass. Solid points denote the stellar-mass measurements of BCGs from Lidman et al., and the solid line denotes the model predictions of De Lucia \& Blaizot (2007). This is a modification of the Lidman et al. plot; here, a value of 0.2 indicates that a BCG has a deficit of $20 \%$ in its stellar mass compared to today. The solid gray region shows the amount of stellar mass that, given the assumptions outlined in the text, would be added to BCGs by the dusty enshrouded star formation seen in this work. The upper boundary is calculated by assuming all of the IR-bright systems detected here are star-forming, while for the lower bound we have removed all possible AGNs (Figure 4) from the analysis. The intention of this plot is to compare the approximate mass growth through star formation with that required by the directly observed mass change (Lidman) and that expected from the models (De Lucia \& Blaizot)

redshift bin-but employ a lower average SFR that incorporates the IR-faint galaxies as well. The upper bound of the area includes all IR galaxies, while the lower bound was computed with the candidate AGNs removed.

Figure 10 is meant only to illustrate the approximate importance of the dust-enshrouded star formation seen here, relative to the amount of mass required by other observations. The stellar-mass determination is crude, and no attempt is made to correct for progenitor bias, as done in Lidman et al. Nevertheless, a simple picture emerges. Out to $z \sim 1$, in situ star formation adds less to the total stellar mass of the BCGs (as a population) than do the dry-merger predictions and is less than required by the mass measurements of Lidman et al. Above $z \sim 1$, however, the situation is less clear. The amount of mass added by star formation rises steeply and begins to approach the mass assembled through dry mergers in the models. At $z \sim 1.5$, both processes would more than double the existing mass of the BCG. More mass measurements of BCGs above $z \sim 1$ are required to determine if there is actual tension between these two models of mass assembly. Clearly both processes could operate and play significant roles in the formation of BCGs; however, their combined effect is constrained by the observed buildup of stellar mass.

\subsection{Comparison to Recent Literature}

The results presented here stem from the first infrared analysis of a large sample of BCGs at $0.2<z<1.8$ They are, however, in solid agreement with complementary studies of other massive galaxies and single clusters. Marchesini et al. (2014) undertook a study of the progenitors of local ultramassive galaxies (UMGs) within the UltraVista Survey using abundance matching techniques. While not explicitly identified with clusters, the stellar mass of $10^{11.8} M_{\odot}$ is similar to the mass of our BCGs $3 \times 10^{10}-7 \times 10^{11}$, and it is likely that the Marchesini et al. population overlaps significantly with the population studied here. They find remarkably consistent results: the progenitors of UMGs remain quiescent until $z \sim$ 1 , beyond which they exhibit extreme levels of dusty star formation of several hundreds of solar masses per year.

Extreme star formation activity has also been measured toward or in the cores of several high-redshift clusters. Santos et al. (2015) report a total SFR of $1875 M_{\odot} \mathrm{yr}^{-1}$ within the central $250 \mathrm{kpc}$ of a $z=1.58$ cluster, distributed over three massive galaxies. Indeed, many groups are now finding the star formation activity within the central regions of $z>1.5$ clusters, though not always the BCG (Tran et al. 2010; Fassbender et al. 2011, 2014; Popesso et al. 2012; Brodwin et al. 2013), is consistent with the level of field activity. In some cases (e.g., Fassbender et al. 2014), this activity is due to an enhancement in galaxy-galaxy mergers within the cluster cores.

\subsection{Star Formation Driver: Dissipative Mergers?}

These results of the previous sections indicate a rapid increase in star formation in the central galaxies of clusters beyond $z \sim 1$, and this in turn suggests an increase in the efficiency of gas deposition onto these systems. In the field, major galaxy mergers appear to be the dominant method of delivering new (and large) reservoirs of gas to galaxies, but this process may be problematic in very high density regions. Galaxies within the cluster environment are subject to ISM removal processes such as ram pressure stripping or strangulation and may be gas deficient (e.g., Gavazzi et al. 2006) by the time they reach the center. As pointed out in McDonald et al. (2012) for the Phoenix Cluster BCG, the prodigious SFRs measured here require the accretion galaxy with a gas mass far in excess $(10-100 \times)$ of that of a typical $(z=0)$ late-type cluster galaxy. Certainly, infalling field galaxies at high redshift will begin their accretion with more gas than at low redshift (Geach et al. 2011), and therefore may be able to retain a larger overall mass of gas during their infall. Indeed, simulations of galaxyICM interaction indicate galaxies may retain substantial fractions of their gas during infall to the cluster center (Jáchym et al. 2007), but the gas content of cluster galaxies beyond $z \sim$ 1 remains unconstrained.

Still, the one high-redshift and $24 \mu \mathrm{m}$ bright galaxy for which we have conducted high spatial resolution imaging with the HST shows clear indications of a merger (Webb et al. 2015). This system, SpARCS1049+56, consists of a long $(66 \mathrm{kpc})$ tidal tail interspersed with clumps along its length. It appears to originate from within the stellar halo of the BCG and exhibits copious amounts of star formation $(\sim 1100$ $M_{\odot} \mathrm{yr}^{-1}$ ). This gas-rich merger would not have been apparent without HST NIR imaging, and thus ruling out this scenario for the rest of the sample is premature. If SpARCS1049+56 is indeed representative of the $z>124 \mu \mathrm{m}$ bright SpARCS sample, then wet gas-rich mergers can occur at the centers of clusters and, indeed, are an important process in building the stellar mass of BCG at early times.

On the other hand, studies of cool-core clusters (Liu et al. 2012; Rawle et al. 2012) reveal a strong correlation between the SFRs of BCGs (limited to below $z \sim 0.5$ ) and their cooling times (as measured in the X-ray). The sample presented here is optical-richness selected (nominally stellar-mass selected), and there is no information on the state of the gas in these systems. 
X-ray studies of the Red-Sequence Cluster Survey (Hicks et al. 2013), which are limited to $z<1$, indicate that perhaps $10 \%$ of optically selected clusters harbor cool-cores. This fraction is enough to account for all IR-detected galaxies in our sample to $z \sim 1$, but does require the number of cool-core clusters to increase substantially at higher redshifts. A detailed comparison of SpARCS optical and X-ray-selected clusters has not yet been made at these redshifts. One SpARCS field (XMM-LSS) has X-ray coverage, and we have compared the clusters found by the SpARCS survey with those identified in Adami et al. (2011) and Willis et al. (2013). At the significance and richness limit of SpARCs, the overlap is primarily restricted to the most X-ray-luminous systems at $z<0.6$, where the IR detection rate is low. Only one common cluster has a BCG detected at $24 \mu \mathrm{m}$, and it is not unusual in its X-ray luminosity. X-ray observations of the SpARCS sample would provide a simple yet clear confirmation of this idea-the $20 \%$ of the sample that is IR-luminous should be cool-core clusters when observed in the X-ray.

\section{SUMMARY, CONCLUSIONS, AND FUTURE WORK}

We investigate the infrared properties of a large sample (535) of BCGs in massive optically selected galaxy clusters over the redshift range $0.2<z<1.8$, using the SWIRE public $24 \mu \mathrm{m}$ imaging and catalogs. We find several important results.

1. We detect $125 / 535$, or $23 \%$, of the BCGs at $24 \mu \mathrm{m}$. The fraction of BCGs detected at $24 \mu \mathrm{m}$ above $100 \mu \mathrm{Jy}$ and above a fixed IR luminosity increases beyond redshift $z \sim$ 1. Below $z<1$, no BCGs show $L_{\mathrm{IR}}>10^{12} L_{\odot}$, whereas above $z>1$, this fraction rises to $20 \%$.

2. An investigation of the Spitzer-IRAC IR colors indicates that the bulk of the IR emission of BCGs is not AGN dominated, but rather is due to dust-enshrouded star formation. Only $7 / 125$ of the $24 \mu \mathrm{m}$ detected BCG sample inhabit the AGN region (defined by Donley et al. 2012) of the IRAC color-color diagram.

3. Assuming no contribution from AGNs to the infrared luminosity, we calculate the SFRs for the BCGs. These range from $\sim 1 M_{\odot} \mathrm{yr}^{-1}$ at low redshift to $\sim 1000 M_{\odot} \mathrm{yr}^{-1}$ at high redshift. At all redshifts, however, the specific SFRs of the BCGs are consistent with those of main-sequence star-forming galaxies of similar mass, and thus the bulk of the BCG population is not starbursting.

4. We argue the the star formation episodes are short lived and below $z \sim 1$ do not contribute more than $10 \%$ to the final total stellar mass of the BCGs. In this case, BCG growth is likely dominated by dry mergers. Above $z \sim 1$, however, the star formation seen here may double the stellar mass of the BCGs and is thus an important, and perhaps dominate, process for stellar-mass growth.

5. The physics driving the increase in star formation above is as yet unconstrained, although the one object for which we have deep and high spatial resolution imaging $($ SpARCS1049+56) shows clear signs of wet merger activity.

Funding for PRIMUS is provided by NSF (AST-0607701, AST-0908246, AST-0908442, AST-0908354) and NASA (Spitzer-1356708, 08-ADP08-0019, NNX09AC95G). T.M.A. W. acknowledges the support of an NSERC Discovery Grant.
Funding for SDSS-III has been provided by the Alfred P. Sloan Foundation, the Participating Institutions, the National Science Foundation, and the U.S. Department of Energy Office of Science. The SDSS-III web site is http://www.sdss3.org/. SDSS-III is managed by the Astrophysical Research Consortium for the Participating Institutions of the SDSS-III Collaboration including the University of Arizona, the Brazilian Participation Group, Brookhaven National Laboratory, Carnegie Mellon University, University of Florida, the French Participation Group, the German Participation Group, Harvard University, the Instituto de Astrofisica de Canarias, the Michigan State/Notre Dame/JINA Participation Group, Johns Hopkins University, Lawrence Berkeley National Laboratory, Max Planck Institute for Astrophysics, Max Planck Institute for Extraterrestrial Physics, New Mexico State University, New York University, Ohio State University, Pennsylvania State University, University of Portsmouth, Princeton University, the Spanish Participation Group, University of Tokyo, University of Utah, Vanderbilt University, University of Virginia, University of Washington, and Yale University. Financial support for this work was provided by NASA through programs GO-13306, GO-13677, GO-13747, GO-13845, and GO-14327 from the Space Telescope Science Institute, which is operated by AURA, Inc., under NASA contract NAS 5-26555.

Facilities: Spitzer Space Telescope (MIPS; IRAC), Magellan (IMACS), CTIO, CFHT

\section{REFERENCES}

Adami, C., Mazure, A., \& Pierre, M. 2011, A\&A, 526, 18

Allen, S. W., Edge, A. C., Fabian, A. C., et al. 1992, MNRAS, 259, 67

Andreon, S., \& Congdon, P. 2014, A\&A, 568, 23

Bell, E. F., McIntosh, D. H., Katz, N., \& Weinberg, M. D. 2003, ApJS, 149,289

Bower, R. G., Benson, A. J., Malbon, R., et al. 2006, MNRAS, 370, 645

Brodwin, M., Stanford, S. A., \& Gonzalez, A. H. 2013, ApJ, 779, 138

Bruzual, G., \& Charlot, S. 2003, MNRAS, 344, 1000

Capozzi, D., Collins, C. A., Stott, J. P., \& Hilton, M. 2012, MNRAS, 419, 282

Cardiel, N., Gorgas, J., \& Aragon-Salamanca, A. 1998, MNRAS, 298, 977

Chary, R., \& Elbaz, D. 2001, ApJ, 556, 562

Coil, A. L. \& PRIMUS TEAM, et al. 2011, ApJ, 741, 8

Collins, C. A. 2009, Natur, 458, 603

Cool, R. J. \& PRIMUS TEAM, et al. 2013, ApJ, 767, 118

Crawford, C. S., Allen, S. W., Ebeling, H., Edge, A. C., \& Fabian, A. C. 1999 MNRAS, 306, 857

Daddi, E., Dickinson, M., Morrison, G., et al. 2007, ApJ, 670, 156

De Lucia, G., \& Blaizot 2007, MNRAS, 375, 2

Demarco, R., Wilson, G., \& Muzzin, A. 2010, ApJ, 711, 1185

Donahue, M., de Messiéresm, G. E., O’Connell, R. W., et al. 2011, ApJ, 732, 40

Donley, J., Koekemoer, A. M., \& Brusa, M. 2012, ApJ, 748, 142

Dressler, A. 1978, ApJ, 223, 765

Edge, A. C. 2001, MNRAS, 328, 762

Edwards, L. O. V., Hudson, M. J., Balogh, M. L., \& Smith, R. J. 2007, MNRAS, 379, 100

Edwards, L. O. V., \& Patton, D. R. 2012, MNRAS, 425, 287

Elbaz, D., Daddi, E., \& Le Borgne, D. 2007, A\&A, 468, 33

Elbaz, D., Dickinson, M., Hwang, H. S., et al. 2011, A\&A, 533, 119

Fang, Y., Lidman, C., \& Davis, T. M. 2015, arXiv:1504.03039

Fassbender, R., Böhringer, H., \& Nastasi, A. 2011, A\&A, 527, 10

Fassbender, R. B., Natasi, A., \& Santos, J. S. 2014, A\&A, 568, 5

Gavazzi, G., O’Neil, K., Boselli, A., \& van Driel, W. 2006, A\&A, 449, 929

Geach, J. E., Simpson, C., Rawlings, S., Read, A. M., \& Watson, M. 2007, MNRAS, 381, 1369

Geach, J. E., Smail, I., \& Moran, S. M. 2011, ApJL, 730, L19

Gladders, M., \& Yee, H. K. C. 2005, ApJS, 157, 1

Grogin, N. A., Kocevski, D. D., \& Faber, S. M. 2011, ApJS, 197, 35

Guo, Q. 2011, MNRAS, 413, 101

Hezaveh, Y., Marrone, D., \& Holder, G. 2012, ApJ, 761, 20 
Hicks, A., Pratt, G. W., \& Donahue, M. 2013, MNRAS, 431, 2542 Hildebrandt, H., Muzzin, A., \& Erben, T. 2011, ApJL, 733, 30

Jáchym, P., Palous, J., Koppin, J., \& Combes, F. 2007, A\&A, 472, 5

Johnstone, R. M., Fabian, A. C., \& Nulsen, P. E. J. 1987, MNRAS, 224, 75

Kennicutt, R. 1998, ApJ, 498, 181

Koekemoer, A. M., Faber, S. M., Ferguson, H. C., et al. 2011, ApJS, 197, 36

Lacy, M., Sajina, A., \& Petric, A. O. 2007, AJ, 133, 186

Lacy, M., Storrie-Lombari, L. J., \& Sajina, A. 2004, ApJS, 154, 166

Le Floc'h, E., Papovich, C., Dole, H., et al. 2005, ApJ, 632, 169

Lidman, C., Suherli, J., \& Muzzin, A. 2012, MNRAS, 427, 550

Lidman, C., Iacobuta, G., \& Bauer, A. E. 2013, MNRAS, 433, 825

Liu, F. S., Mao, S., \& Meng, X. M. 2012, MNRAS, 423, 422

Lonsdale, C., Polletta, M., \& Surace, J. 2004, ApJS, 154, 54

Marchesini, D., Muzzin, A., \& Stefanon, M. 2014, ApJ, 794, 65

McDonald, M., Benson, B., Veilleux, S., Bautz, M. W., \& Reichardt, C. L. 2013, ApJL, 765, 37

McDonald, M., Bayliss, M., \& Benson, B. A. 2012, Natur, 488, 349

McNamara, B., \& Nulsen, P. E. J. 2007, ARA\&A, 45, 117

McNamara, B., \& O'Connell, R. W. 1992, ApJ, 393, 579

Murphy, E. J., Chary, R.-R., Alexander, D. M., et al. 2009, ApJ, 698, 1380

Muzzin, A., Wilson, G., \& Yee, H. K. C. 2009, ApJ, 698, 1934

Muzzin, A., Wilson, G., \& Yee, H. K. C. 2012, ApJ, 746, 188

Nordon, R., Lutz, D., \& Genzel, R. 2012, ApJ, 75, 182

O’Dea, K. P., Quillen, A. C., O'Dea, C. P., et al. 2010, ApJ, 719, 1619

Oemler, A., Jr. 1976, ApJ, 209, 693
Popesso, P., Biviano, A., Rodighiero, G., et al. 2012, A\&A, 537, 58

Rafferty, D. A., McNamara, B. R., \& Nulsen, P. E. J. 2008, ApJ, 687, 899

Raue, M., \& Meyer, M. 2012, MNRAS, 426, 1097

Rawle, T. D., Edge, A. C., \& Egami, E. 2012, ApJ, 747, 29

Rodighiero, G., Cimatti, A., \& Gruppioni, C. 2010, A\&A, 518, L25

Rowan-Robinson, M., Gonzalez-Solares, E., Vaccari, M., \& Marchetti, L. 2013, MNRAS, 428, 1958

Rowan-Robinson, M., Babbedge, T., \& Oliver, S. 2008, MNRAS, 386, 697

Sajina, A., Lacy, M., \& Scott, D. 2005, ApJ, 621, 256

Santos, J. S., Altieri, B., \& Valtchanov, I. 2015, MNRAS, 447, 65

Santos, J. S., Rosati, P., \& Gobat, R. 2009, A\&A, 501, 49

Schombert, J. M. 1986, ApJS, 60, 603

Stern, D., Eisenhardt, P., \& Gorjian, V. 2005, ApJ, 631, 163

Stott, J. P., Collins, C. A., Burke, C., Hamilton-Moris, V., \& Smith, G. P. 2011, MNRAS, 414, 445

Tran, K.-V. H., Papovich, C., Saintonge, A., et al. 2010, ApJL, 719, 126

Tremain, S. D., \& Richstone, D. O. 1977, ApJ, 212, 311

Voit, G. M., Cavagnolo, K. W., Donahue, M., et al. 2008, ApJL, 681, 5

Webb, T., Noble, A., \& DeGroot, A. 2015, ApJ, 809, 173

Wen, Z. L., Han, J. L., \& Liu, F. S. 2010, ApJS, 187, 272

Wen, Z. L., Han, J. L., \& Liu, F. S. 2012, ApJS, 199, 34

Wenger, M., Ochsenbein, F., \& Egret, D. 2000, A\&AS, 143, 9

Willis, J. P., Clerc, N., \& Bremer, M. N. 2013, MNRAS, 430, 134

Wilman, R. J., Edge, A. C., \& Swinbank, A. M. 2006, MNRAS, 371, 93

Wilson, G., Muzzin, A., \& Yee, H. K. C. 2009, ApJ, 698, 1943 